\title{
Skin Response Supplemental Qualifiers Dataset
}

National Cancer Institute

\section{Source}

National Cancer Institute. Skin Response Supplemental Qualifiers Dataset. NCI

Thesaurus. Code C147250.

A dataset containing supplemental information, specifically non-standard variables, to parent records in the skin response domain. 